\title{
Abstracts From the 6th Chronic Hypoxia Symposium, October 8-15, 2016, La Paz, Bolivia
}

\section{Dedicated to the Late Prof. Dr. Gustavo Zubieta-Castillo}

Every two years the Chronic Hypoxia Symposium is carried out in order to gather scientists from around the globe to present their work and discuss aspects of chronic exposure to hypoxia. The first three symposia, The Effect of Chronic Hypoxia on Diseases at High Altitude, were held in La Paz, Bolivia at $3510 \mathrm{~m}$, the "shrine of hypoxia" and location of the High Altitude Pulmonary and Pathology Institute (IPPA), the first high-altitude clinic in the world, created on July 9th, 1970 by the late Prof. Dr. Gustavo Zubieta-Castillo Sr. The fourth symposium was carried out jointly with the Global Hypoxia Summit at the Defense Institute of Physiology and Allied Sciences (DIPAS) in Delhi, India. The fifth and this one, the sixth, returned to La Paz. It was an itinerant meeting, starting the first day in $\mathrm{La} \mathrm{Paz}$ at $3500 \mathrm{~m}$, the second in the Tiahuanacu ruins $(3800 \mathrm{~m})$, the third day at Lake Titicaca $(3800 \mathrm{~m})$, the highest navigable lake in the world, and the fourth day in Chacaltaya at $5300 \mathrm{~m}$, where the Glass Pyramid Laboratory, the highest laboratory in the world, is located. The spirit of this symposium is an open mind to different concepts about exposure and life under chronic hypoxia, with a criterion that adaptation to chronic hypoxia is successful and often misunderstood.

\section{The Road to "Defeating Hypoxia" is a Timely Exposure to Chronic Hypoxia}

Gustavo Zubieta-Calleja, MD

High Altitude Pulmonary and Pathology Institute (IPPA), La Paz, Bolivia

Acute exposure to hypobaric hypoxia can give rise to acute mountain sickness, and in some severe cases, high altitude pulmonary edema (HAPE) and high altitude cerebral edema (HACE), that can sometimes lead to the loss of life. However, once the acute phase evolves from gradual adaptation to a fixed "chronic hypoxia" altitude, following the Adaptation to High Altitude Formula (adaptation to high altitude $=$ time/altitude), the organism does remarkably well. Life under chronic hypoxia, where an optimal hematocrit is reached for every fixed altitude, following a logarithmic curve in relation to time, is practically as normal as that at sea level. The cities of La Paz (3100-4100 m) and El Alto $(4100 \mathrm{~m})$ stand as living proof of this. Over 2 million inhabitants carry out perfectly normal lives, undisturbed by hypoxia and most even unaware of its existence. The lungs increase in volume, the right heart is mildly hypertrophied secondary to a normal adaptive pulmonary hypertension (relative to sea level). All the cells of the organism likewise adapt to a lower arterial oxygen arterial partial pressure $\left(\mathrm{P}_{\mathrm{a}} \mathrm{O}_{2}\right)$ but also to a lower arterial partial pressure of carbon dioxide $\left(\mathrm{P}_{\mathrm{a}} \mathrm{CO}_{2}\right)$, an essential component that linked to an increased hemoglobin explain the paradox of increased "tolerance to hypoxia" at high altitude. The higher the altitude, the more tolerance to hypoxia.

Life under chronic hypoxia is not only tolerable, but also is, in fact, favorable to improve or treat many pathological conditions such as asthma, coronary artery disease, obesity and even giving rise to improved longevity.

"Every living being, according to the genetic buildup-that determines their species, race and sex-has an optimal form of adaptation and the proof lies in a successful reproduction and survival at any altitude." - Prof. Dr. Gustavo Zubieta-Calleja

\section{Interindividual Differences in the Adaptive Response} of the Lung to Hypoxia

Giuseppe Miserocchi, MD

Former Professor of Physiology and Biophysics, Università Milano-Bicocca, Italia
The adaptive response of the lung to hypoxia is dominated by the risk of potential development of lung edema on one side, and, on the other, by the need to develop an adaptive response allowing to preserve the oxygen diffusion-transport function. The edemagenic condition relates to the increased cardiac output and microvascular permeability. The rigidity of the extravascular interstitial matrix represents the main mechanism to resist edema formation: due to this peculiar feature, the increase in microvascular filtration leads to a corresponding remarkable increase in interstitial pressure that strongly buffers filtration. A further antiedemagenic mechanism is based on blood flow restriction to the septal microcirculation. This occurs by active vasoconstriction in the regions where edema tends to develop. A further cause of blood flow limitation in the septal capillary bed is due to the increase in the interstitial pressure that decreases the patency of the capillaries. The blood flow limitation in the septal capillary bed leads to increased perfusion of the corner vessels running at the edge of the alveoli; the overall increase in blood flow vascular resistance is matched by an increase in pulmonary artery pressure. A surprising finding is that in about $55 \%$ of subjects, $D L_{C O}$ increased in hypoxia (exposure to $3500 \mathrm{~m}$ ), reflecting an increase in conductance of the air blood barrier $D m$ that largely overruled the decrease in in capillary blood volume $(V c)$. In about $25 \%$ subjects $D L_{C O}$ remained unchanged in hypoxia while it decreased in $15 \%$. We will explore how individual oxygen diffusion-transport features influence this response.

\section{Interindividual Differences Concerning the Oxygen Diffusion-Transport Function in the Lung}

Giuseppe Miserocchi, MD

Former Professor of Physiology and Biophysics, Università MilanoBicocca, Italia

The oxygen diffusion/transport function is accomplished at the level of the airblood barrier. Lung diffusion $\left(\mathrm{DL}_{\mathrm{CO}}\right)$ is currently measured using carbon monoxide as a tracer gas that is not diffusion limited. Subcomponents of $\mathrm{DL}_{\mathrm{CO}}$ can also be derived representing surface membrane diffusivity $(\mathrm{Dm})$ and volume of blood present in the lung capillary, allowing oxygen-Hb binding ( $\mathrm{Vc}$ ). Subjects differed substantially in terms of $\mathrm{DL}_{\mathrm{CO}}, \mathrm{Dm}$, and $\mathrm{Vc}$. We attempted to estimate diffusion limitation in subjects exposed to $3850 \mathrm{~m}$ by determining the decrease of the $\mathrm{DO}_{2} / \beta \dot{Q}$ individual ratio, the so-called "equilibration index" (Piiper and Scheid, Respir Physiol, 1981), where $\mathrm{DO}_{2}$, the oxygen diffusion capacity, was calculated as $\mathrm{DL}_{\mathrm{CO}} \cdot 1.23$, Q was cardiac output, and $\beta$ was the hemoglobin binding capacity for $\mathrm{O}_{2}$. A decrease in the "equilibration index," potentially leading to diffusion limitation, was found in all subjects on exposure to hypoxia, yet, the lack of equilibration did not exceed 0.25 ( 0 corresponding to perfect equilibration, namely, no alveolo-capillary $\mathrm{O}_{2}$ pressure difference) Subjects having a high equilibration index at sea level due to high $\mathrm{DO}_{2}$, high Dm, and a low heart rate have an advantage to prevent the fall of the equilibration index below 2 , a critical value below which diffusion limitation occurs.

\section{Potential Application of Cerium Dioxide Nanoparticles for Acute Pneumonia Treatment}

Z. Serebrovska, $\mathrm{PhD}^{1}$; V. Dosenko, $\mathrm{MD}, \mathrm{PhD}^{1}$; A. Shysh, MS, $\mathrm{PhD}^{1}$; S. Pavlovich, $\mathrm{PhD}^{1}$; A. Dorovskych, $\mathrm{PhD}^{2}$; V. Lysenko, $\mathrm{PhD}^{3}$; V. Tertykh, $\mathrm{PhD}^{3}$; J. Bolbuckh, $\mathrm{PhD}^{3} ;$ V. Portnichenko, $\mathrm{MD}, \mathrm{PhD}^{3}$

${ }^{1}$ Bogomoletz Institute of Physiology, National Academy of Sciences, Ukraine, ${ }^{2}$ Integrative Medicine Clinic, "SmartMed," Kiev, Ukraine,

${ }^{3}$ Lashkariov Institute of Semiconductor Physics, Kiev, Ukraine 
Background.-Cerium dioxide nanoparticles $\left(\mathrm{CeO}_{2} \mathrm{NP}\right)$ have antioxidant properties and also can alter membrane potential in mitochondria influencing cell respiration. In this study we check the idea that $\mathrm{CeO}_{2} \mathrm{NP}$ adsorbed on the silicon base has counter inflammatory, antioxidant and metabolic effects and can be possibly applied in pneumonia treatment.

Methods.- Thirty-six Wistar male rats, 5 months old, 250-290 g were divided in five groups: 1) intact animals; 2) i/p saline injection; 3) $\mathrm{CeO}_{2} \mathrm{NP}$ treatment; 4) experimental pneumonia (i/p LPS injection, $1 \mathrm{mg} / \mathrm{kg}$ ); 5) experimental pneumonia treated by $\mathrm{CeO}_{2} \mathrm{NP}(2.5 \mathrm{mg} / \mathrm{kg})$. Pulmonary ventilation and gas exchange were measured four times: before and 1,3 and $24 \mathrm{~h}$ after LPS injection. The mRNA of TNF- $\alpha$, Il- 6 , and CxCL3 were determined by RT-PCR. ROSgeneration in blood plasma and lung tissue homogenate was measured by means of lucigenin- and luminol-enhanced chemiluminescence.

Results.-Endotoxaemia in the acute phase was associated with essential inhibition of metabolism: gradual decrease of $\mathrm{V}_{\mathrm{E}}$ (by $72 \%$ ) and $\mathrm{VO}_{2}$ (by 62\%) 24 hour after LPS injection; pathological changes in lung morphology; enhanced expression of CxCL3 (by 68\%); increase of ROS generation in blood (by 660\%) and lungs (in $33 \%$ ).

LPS-injected rats treated with $\mathrm{CeO}_{2} \mathrm{NP}$ did not demonstrate any $\mathrm{V}_{\mathrm{E}}$ and $\mathrm{VO}_{2}$ reduction 1 hour after LPS-injection and increase of $\mathrm{V}_{\mathrm{E}}$ (by 25\%) and $\mathrm{VO}_{2}$ (by $20 \%) 3$ hours after injection; this effect remained 24 hours after injection.

It should be noted that $\mathrm{CeO}_{2} \mathrm{NP}$ treatment of control rats increased $\mathrm{V}_{\mathrm{E}}$ by $20 \%$ and $\mathrm{VO}_{2}$ by $45 \%$ in comparison with first measurement after each introduction of the substance.

Treatment of LPS-injected rats with $\mathrm{CeO}_{2} \mathrm{NP}$ has led to diminishing in lung morphology pathological deviations; significant decrease of the expression of inflammatory markers: CxCL33 (in 7.1 times, p $<0.01$ ), Il-6 (in 3.5 times, $\mathrm{p}<0.05$ ) and TNF- $\alpha$ (in 2 times, $\mathrm{p}=0.05$ ); significant reducing of ROS generation in blood (in 2.7 times, $\mathrm{p}<0.05$ ), and lung tissue homogenate (in 1.3 times $\mathrm{p}<0.05)$.

Conclusions.- $\mathrm{CeO}_{2}$ NP significantly reduces morphological and functional pulmonary damage in course of experimental acute pneumonia. Discussing mechanism of this effect we propose two suggestions. First: $\mathrm{CeO}_{2} \mathrm{NP}$ is powerful antioxidant, which protects lung tissue and, possibly, inhibits synthesis of cytokines. Second: treatment with $\mathrm{CeO}_{2} \mathrm{NP}$ significantly accelerate metabolism and gives additional vigor to cells suffering with pneumonia.

\section{Life in High-Altitude Conditions}

Thuppil Venkatesh, PhD, FACBI

Profesor Emeritus, Dept of Biochemistry, St. John's Medical College, Bangalore, India

I have always accepted and endorsed both Prof. Dr. Gustavo Zubieta-Castillo (Sr) and Prof. Dr. Gustavo Zubieta-Calleja (Jr) whenever they mentioned that life is possible on top of Mt Everest. I never doubted. I have a lot of supporting evidence which I wish to share with the rest of the world, during this meeting at very high altitude in Chacaltaya $(5230 \mathrm{~m})$ where I deliver my talk for the second time. Life does exist at extreme conditions of heat, pressure, temperature, radiation etc. We have functional life at the deepest part of the sea and at very high altitude. Life started at the bottom of the sea and birds fly for long hours at very high altitudes during their migration. People migrated to live and reproduce at high altitudes. The secret of life adaptation is not well understood. Scientists have designed their studies to find out the problems faced during their adaptation. Limited efforts are made to realize how life has sustained for long periods of time at extreme conditions. The most common household pests, cockroaches, have survived severe radiation, heat, and cold and even toxic pesticides, to which they adapt and can therefore not be eliminated. Plants which normally grow at a particular rate under normal conditions, are found growing at a higher rate when their life is threatened, as noted in young mango plants when pruned, they flower and yield fruit earlier. We are aware of the longevity in plants when they are bonsaied. Similar life prolongation is noted in humans and animals under hypoxic conditions. Longevity of life under hypoxic conditions is well established and it seems evident that high altitude residents have a healthy form of life. Lowlanders under hypoxic conditions and highlanders under hyperbaric conditions, as presented by Prof. Dr. Gustavo Zubieta-Calleja, will have initial problems (peripheral edema, somnolence and other changes), which get adjusted. On long stays, with generations living in those environments, there will be a genetic adaptation, which is applicable to all forms of life. Physical and mental performance improves at higher altitudes. Hypoxia makes other dormant parts of the human brain function, and hence great philosophers and religious giants frequently visited high altitudes. When these environments are altered by any manmade developments, it hampers the process of natural adaptation. Many toxins are now found causing environmental damage at various levels. This is evidenced by the unreasonable usage of medication. Living in harmony with nature seems to be the ultimate solution. The effect seems to be more evident on life at high altitudes. Let us preserve high altitude conditions for better health, for quality longevity. Let the dream of the Parvatha Guru (Mountain Guru), Gustavo Zubieta-Castillo Sr., come true.

\section{Rearrangement of Neuroendocrine Systems in the Mountains}

Sadykova Gulnura, MD; Dzhunusova Gulnara, MD

Institute of Mountain Physiology National Academy of Sciences, Kyrgyz Republic

The aim of this research was to study the functional state of the anterior pituitary, thyroid, adrenal, and reproductive glands and their relationship with chronic exposure to high altitude. Studies were conducted in high altitudes inhabitants of the Tien-Shan $(1200 \mathrm{~m}, 2800 \mathrm{~m}, 3600 \mathrm{~m})$. Adrenocorticotropic hormone (ACTH), cortisol, catecholamines, thyroid, and sex hormones were determined by immunosorbent and spectrofluorometric methods. Normal hormone levels for sea level inhabitants were used for comparison.

Increased cortisol levels in mountaineers $(\mathrm{M})(2800 \mathrm{~m})$ may reflect a reduction in reserve capacity of the pituitary-adrenocortical system and the formation of their chronic condition or incomplete adaptation, or the continuing adaptation to the environment in a number of successive generations. Reduced activity of the pituitary-adrenocortical system in high altitude residents (HAR) at $3600 \mathrm{~m}$ is a consequence of the harsh weather conditions, narrowing the boundaries of the organism's adaptation and facilitating economical and appropriate regulation.

High altitude residents at $2800 \mathrm{~m}$ and $3600 \mathrm{~m}$ (when compared to the normal hormone levels and data of low mountain residents at $1200 \mathrm{~m}$ ), presented a predominance of the mediator activity of the hormone sympathetic-adrenal system, showing an increased secretion of norepinephrine and dopamine, and a decreased secretion of epinephrine. Increased levels of norepinephrine are associated with its decreased metabolism and reserves, providing constant catecholamines that create the preconditions for a stable and long-term work of the body, providing an adequate state of physiological function. Feeding habits (prevalence of carbohydrates and fats in food $\mathrm{M}$ and HAR) also lead to the activation secretion of norepinephrine, which leads to intensive use of the biological mediator precursor, dopamine, as evidenced by its high level in residents at both altitudes. This change in optimum reserve of catecholamines promotes an accelerated restoration of catecholamine levels.

Prolonged exposure to high altitude hypoxia induces contrasting changes in thyroid hormone levels. The mountaineers $(2800 \mathrm{~m})$ are found close to the low values of normal TSH levels, whereas thyroid hormones tend to increase. The increase in T3 and T4 levels, while maintaining the basic concentration of TSH or small hyperthyroidism, seem necessary to adapt to the extreme conditions of high mountains. Increased levels of $\mathrm{T}_{4}, \mathrm{TSH}$ and norepinephrine, with a decreased secretion of $\mathrm{T}_{3}$ in HAR $(3600 \mathrm{~m})$, reflect an additional reaction on the PTS factor constant exposure to cold in the mountains. Low values of $\mathrm{T} 3$ without changes in $\mathrm{TSH}$ and $\mathrm{T}_{4}$ levels are explained by the influence of cold

The detected levels of testosterone in male HAR $(3600 \mathrm{~m})$ show the adverse effects of environmental factors, and reduced levels of progesterone (89\%) indicate a hard endocrine function of women M $(2800 \mathrm{~m})$, on the mobilization of the strategic reserve endocrine system. However, the decreased functional activity of the sex glands does not exceed the boundaries of conventional indicators, which are a reflection of the body's resistance.

Conclusion: Features of endocrine systems in high-population of Tien-Shan include reduced activity of the pituitary-adrenocortical system in the organism, and economical operation, which allows for normal life, work and heavy physical activities in the specific conditions of the mountains. The parameters of endocrine status should be assessed only in relation to normal population values.

Why is it Not Possible to Predict, Through Tests at Sea Level, Who Will Develop AMS?

Gustavo Zubieta-Calleja, MD; Natalia Zubieta DeUrioste

High Altitude Pulmonary and Pathology Institute (IPPA), La Paz, Bolivia 